\title{
Retraction Note: Texture-guided volumetric deformation and visualization using 3D moving least squares
}

\author{
Xin Zhao · Bo Li · Lei Wang • Arie Kaufman
}

Published online: 3 July 2014

(C) Springer-Verlag Berlin Heidelberg 2014

\section{Retraction to: Vis Comput (2012) 28:193-204 \\ DOI 10.1007/s00371-011-0635-2}

The authors of this paper requested on November 7, 2013 that this paper will be retracted from The Visual Computer, following an allegation that the paper has used images from some other articles.

- Volume Deformation via Scattered Data Interpolation. Carlos D. Correa, Deborah Silver, and Min Chen. 2007. In Proceedings of the Sixth Eurographics/Ieee VGTC conference on Volume Graphics (VG'07), Hans-Christian Hege and Raghu Machiraju (Eds.). Eurographics Association, Aire-la-Ville, Switzerland, Switzerland, 91-98. doi:10.2312/VG/VG07/091-098

- Feature Aligned Volume Manipulation for Illustration and Visualization. C Correa, D Silver, M Chen. Visualization and Computer Graphics, IEEE Transactions on 12 (5), 1069-1076, 2006

- Visualizing what lies inside. CD Correa. ACM SIGGRAPH Computer Graphics 43 (2), 5, 2009.

The online version of the original article can be found under doi:10.1007/s00371-011-0635-2.

X. Zhao $(\bowtie) \cdot$ B. Li $\cdot$ L. Wang · A. Kaufman

Computer Science Department, Stony Brook University,

Stony Brook, NY 11794, USA

e-mail: xinzhao@cs.sunysb.edu

B. $\mathrm{Li}$

e-mail: bli@cs.stonybrook.edu

L. Wang

e-mail: leiwang1@cs.stonybrook.edu

A. Kaufman

e-mail: ari@cs.stonybrook.edu
Subsequently, the first author Xin Zhao admitted that she has indeed used some images from other articles without acknowledging or disclosing what she did, giving the wrong impression that the figures are the product of her algorithm. All the other co-authors have not been aware of and have not been involved with this misconduct. 